\title{
Estados de cosas y relaciones de fundación
}

\author{
José Tomás Alvarado*
}

Resumen: un estado de cosas es una estructura no-mereológica compuesta por universales, objetos y tiempos. En principio, un estado de cosas no está fundado en sus componentes, pues tales componentes podrían existir, pero no el estado de cosas que componen. La introducción de otras relaciones de 'instanciación' no mejoran la situación, si tales relaciones son universales. Un estado de cosas, sin embargo, sí está fundado en un universal, uno o varios objetos, un tiempo $y$ un tropo que, esencialmente, es la instanciación de tal universal en tal objeto, o en tales objetos, en tal tiempo. Si los estados de cosas están fundados en sus constituyentes, entonces hay una razón para admitir tropos. Finalmente, se discuten varias razones por los que los estados de cosas deben estar fundados.

Palabras clave: estado de cosas, universales, tropos, fundación.

\begin{abstract}
: a state of affairs is a non-mereological structure composed by universals, objects and times. In principle, a state of affairs is not grounded in its components, for those components could exist, but not the state of affairs that they compose. The introduction of other relations of 'instantiation' doesn't improve the situation, if those relations are universal. A state of affairs, though, is grounded on a universal, object or objects, time and a trope that, essentially, is the instantiation of that universal in that object, or in those objects, in that time. If states of affairs are grounded in their constituents, then there is reason to admit tropes. Finally, several reasons by which states of affairs should be grounded are discussed.
\end{abstract}

Key words: state of affairs, universals, tropes, grounding.

\footnotetext{
* Licenciado en Derecho por la Pontificia Universidad Católica de Chile y Doctor en Filosofía por la Universidad de Navarra (España). Actualmente es Profesor Asistente en la Pontificia Universidad Católica de Chile. Sus intereses de investigación están vinculados a la metafísica analítica, la filosofía del lenguaje y la epistemología. Dirección electrónica: jose.tomas.alvarado@gmail.com
} 
Un "estado de cosas" (state of affairs, Sachverbalt) ha sido concebido usualmente como el hecho de instanciar o ejemplificar un objeto (u objetos) una propiedad (o relación) en un instante de tiempo no necesariamente puntual. Un estado de cosas es, entonces, una entidad compleja constituida por otras que -en principio- deberían verse como más 'básicas' que él. Su existencia parece 'depender' de o 'estar fundada en' la existencia de propiedades -sean o no relacionales-, objetos y tiempos. Estas afirmaciones, aunque parezcan razonables, resultan todavía demasiado imprecisas. En este trabajo se pretende atender con más exactitud al modo en que ese estado de cosas puede decirse 'fundado' en tales constituyentes. La relación de 'fundación' (grounding) ha sido objeto de gran atención en los últimos años (cf. Correia y Schnieder, 2012; Schnieder, 2011; Fine, 2010, 2012a, $2012 \mathrm{~b}$ entre otros). Hay una variedad de diferentes conceptos que podrían ser denominados con justicia como relaciones de 'fundación'. Atender a estas diferentes alternativas será útil para comprender el modo de dependencia que pueda ser postulado entre un estado de cosas y sus constituyentes. Una mayor claridad acerca de las relaciones de fundación que puedan ser descritas entre un estado de cosas y sus constituyentes permitirá volver a considerar el famoso regreso de Bradley (cf. Bradley, 1897, 18) por el que se ha sostenido que los estados de cosas -como entidades complejas constituidas por objetos y propiedades- no son inteligibles. La evaluación de las consecuencias que deban sacarse de la situación teórica generada por este argumento podrá ser revisada.

Un 'estado de cosas', tal como se había indicado, será tomado aquí como una complexión de universales, objetos y tiempos. Por supuesto, de un modo general, uno podría sostener que cualquier entidad que funcione como un truthmaker puede contar como 'estado de cosas', en algún sentido de la expresión. Los problemas sistemáticos que están siendo considerados aquí, sin embargo, surgen sólo en ontologías donde se postulan universales. Un universal es una propiedad numéricamente diferente del objeto u objetos en donde puede estar instanciado y que, por su naturaleza, puede encontrarse ejemplificado en una pluralidad de objetos al mismo tiempo. Un universal puede ser monádico o poliádico para $n$ argumentos. En el segundo caso se trata de una relación. En lo que sigue, por generalidad, se supondrá que se trata de universales relacionales $n$-ádicos, pero lo que se dice respecto de universales relacionales vale también para universales monádicos. En ontologías de universales la 'instanciación' del universal 
requiere postular particulares con los que se conforma una estructura. ${ }^{1}$ Esta estructura es lo que se denomina aquí un "estado de cosas". En lo que sigue, el estado de cosas de estar instanciado el universal $U$ en los objetos $x_{1}$, $x_{2}, \ldots, x_{\mathrm{n}}$ en el instante de tiempo no necesariamente puntual $t$ se designará como $\left[\begin{array}{llll}U x_{1} x_{2} & \ldots & x_{n}\end{array}\right]$. Las condiciones de identidad de un estado de cosas vienen dadas por el siguiente principio:

$$
\text { (1) } \begin{aligned}
\left(\left[U_{1} x_{1}, x_{2}, \ldots, x_{\mathrm{n}} t_{\mathrm{n}}\right]=\left[U_{2} x_{\mathrm{m}}, x_{\mathrm{m}+1}, \ldots, x_{\mathrm{m}+\mathrm{i}} t_{2}\right]\right) \leftrightarrow\left(\left(U_{1}=U_{2}\right) \wedge\left(x_{1}=x_{\mathrm{m}}\right)\right. \\
\left.\wedge\left(x_{2}=x_{\mathrm{m}+1}\right) \wedge \ldots \wedge\left(x_{\mathrm{n}}=x_{\mathrm{m}+\mathrm{i}}\right) \wedge\left(t_{1}=t_{2}\right)\right)
\end{aligned}
$$

Esto es, si dos estados de cosas son la instanciación del mismo universal, en los mismos objetos, en el mismo instante de tiempo, entonces se trata del mismo estado de cosas. Se ha sostenido que la forma de estructuración de un estado de cosas -entendido de este modo- no es mereológica, ${ }^{2}$ pues dos estados de cosas con exactamente los mismos componentes podrían ser diferentes. Supóngase, en efecto, un universal relacional diádico asimétrico $R$. Entonces, no parecen ser idénticos los estados de cosas $\left[R x_{1} x_{2} t\right.$ y $\left[R x_{2} x_{1} A\right.$. Si Tristán ama a Isolda, por ejemplo, no se sigue que Isolda ama a Tristán. El estado de cosas de amar Tristán a Isolda no es el mismo estado de cosas de amar Isolda a Tristán. Estados de cosas entendidos del modo indicado hacen surgir la cuestión acerca de cómo está o podría estar un estado de cosas 'fundado' en sus constituyentes, o cómo es

\footnotetext{
${ }^{1}$ Una alternativa sistemática no considerada aquí es la de teorías donde los objetos particulares son cúmulos de universales co-presentes entre sí. Si esta alternativa fuese viable, una ontología uni-categorial, en donde sólo se postulen universales, sería suficiente para generar estados de cosas. Esta alternativa ha parecido, sin embargo, bastante poco verosímil. Por una parte, una concepción de este estilo requeriría aceptar el principio de la identidad de los indiscernibles, pero este principio -salvo en formulaciones triviales- parece falso. Por otra parte, los hechos primitivos de 'co-presencia' entre universales son extremadamente sospechosos en una ontología de este tipo. Cf. Armstrong, 1978a, 89-101; 1989, 59-74.

${ }^{2}$ En lo que sigue, por "mereología" se entenderá la mereología extensional estándar, que puede ser caracterizada por estos tres postulados: (i) transitividad: si $x$ es parte de una parte de $y$, entonces $x$ es parte de $y$; (ii) composición irrestricta: siempre que existan ciertas cosas, entonces existirá la fusión de esas cosas; y (iii) unicidad de composición: nunca las mismas cosas tienen fusiones diferentes (cf. Lewis, 1991, 74; también para una presentación más general, Simons, 1987, 1-45).
} 
que un estado de cosas es o podría ser 'dependiente ontológicamente' de sus componentes.

En lo que sigue, por lo tanto, se va a considerar primero de un modo general las características de la relación de fundación ontológica y sus conexiones con otras nociones cercanas, tal como la de 'dependencia ontológica'. En segundo lugar, se va a considerar de manera más detenida la cuestión de la fundación de los estados de cosas en sus componentes y el regreso de Bradley. En tercer lugar, se va a sostener que si se postula un tropo que sea esencialmente la instanciación de un universal en uno o varios objetos en un tiempo, entonces hay fundación del estado de cosas en sus componentes y se evita el regreso de Bradley. Por último, se discutirán algunos motivos por los que debería sostenerse que los estados de cosas deben estar fundados.

\section{Fundación y dependencia ontológica}

Un estado de cosas entendido del modo que se ha explicado tiene una forma de estructuración peculiar, tal como se ha visto. No parece consistir en una mera fusión mereológica de universales, objetos y tiempos. Tampoco parece consistir en un conjunto de universales, objetos y tiempos. Hay algo 'adicional' en un estado de cosas. Con todo, un estado de cosas debe entenderse como 'dependiente' ontológicamente de sus componentes. Las condiciones de identidad de un estado de cosas, tal como se enuncian en el principio (1) se heredan de las condiciones de identidad de universales, objetos y tiempos. Conviene, entonces, considerar con más detención las nociones de 'fundación' (grounding) y 'dependencia' para luego considerar el tipo de conexión sistemática existente entre un estado de cosas y sus componentes.

Aunque el interés por el concepto ontológico de 'fundación' es reciente, la cuestión de si un tipo de hechos se encuentran o no 'fundados' en otros ha acompañado la reflexión filosófica desde sus comienzos (cf. Correia y Schnieder, 2012b, 2-10; Audi, 2012). Se ha discutido, por ejemplo, si acaso el carácter piadoso de una acción se encuentra fundada en el amor de los dioses, o si es que -al revés- son los hechos acerca del amor de los dioses los que están fundados en el carácter objetivamente piadoso de una acción (cf. Platón, Eutifrón, 6d). Se ha considerado si acaso los estados mentales se encuentran o no fundados en los estados físicos del encéfalo. Se ha considerado si acaso los hechos morales están fundados en hechos 
no-morales, o si los hechos estéticos están o no fundados en hechos no-estéticos. Se ha discutido si acaso las propiedades disposicionales están o no fundadas en propiedades categóricas. Se ha discutido acerca de si la verdad de las proposiciones está o no siempre fundada en entidades del mundo que funcionen como sus truthmakers. Estos ejemplos podrían ser multiplicados ad nauseam. En casi cada área de reflexión filosófica han surgido cuestiones de fundación. Es más, se ha sostenido recientemente que el objeto de la metafísica como disciplina intelectual es la especificación de relaciones de fundación entre categorías de entidades (cf. Schaffer, 2009). Esto es, la metafísica no versaría acerca de si hay o no entidades de ciertas categorías, sino acerca de qué está fundado en qué. Por supuesto, estas cuestiones de fundación no han aparecido específicamente bajo el nombre de "fundación" (grounding), sino bajo otros rótulos. En los días de gloria del giro lingüístico se suponía que cuestiones de este tipo serían resueltas por la 'traducción' de un tipo de discurso a otro. Después se han formulado más bien en términos de "reducción", "eliminación" o "superveniencia". Cada uno de estos conceptos tiene sus dificultades que no serán discutidas aquí. ${ }^{3}$ Lo que es peculiar de los problemas de 'fundación' es que tienen que ver con ciertas relaciones de 'determinación' entre tipos de entidades, o con ciertas formas de 'explicación' no causal entre tipos de entidades, que han sido una y otra vez objeto de la atención del filósofo.

Uno podría estar inclinado a pensar que esta relación podría ser analizada en términos modales. Se ha sostenido de manera convincente, sin embargo, que no lo es (cf. en especial, Fine, 1994, 1995). Nuestra intuición es que, por ejemplo, o bien los hechos sobre el carácter piadoso de los actos está fundado en el amor de los dioses, o bien son los hechos acerca del amor de los dioses los que están fundados en el carácter objetivamente piadoso de los actos. Desde un punto de vista modal, sin embargo, no hay ningún

\footnotetext{
${ }^{3}$ Por ejemplo, la 'eliminación' de los hechos A por los hechos B implica que no hay hechos A. La 'reducción' de los hechos A a los hechos B requiere postular que, por ejemplo, las propiedades del tipo característico para los hechos A son idénticas a ciertas propiedades características de los hechos B. Pero la identidad es simétrica y reflexiva. La superveniencia de los hechos $\mathrm{A}$ a los hechos $\mathrm{B}$, por otro lado, no es necesariamente asimétrica. Es compatible con la identidad de los hechos A con los hechos $\mathrm{B}$, y también es compatible con una conexión causal necesaria entre los hechos del tipo A con los hechos de tipo B.
} 
motivo para hacer este contraste, pues es necesario que algo es piadoso si y sólo si es amado por los dioses. Tenemos también intuiciones acerca de la relación entre un objeto y el conjunto singleton cuyo único elemento es ese objeto. Suponemos, en general, que el objeto $a$ tiene cierto tipo de 'prioridad' respecto del conjunto $\{a\}$. Desde un punto de vista modal, sin embargo, no es posible hacer este contraste, pues es necesario que $a$ existe si y sólo si $\{a\}$ existe. Pero hay más problemas. Supóngase que uno quisiese analizar la relación de fundación por una implicación estricta o una bi-implicación estricta. ${ }^{4}$ Uno podría sostener, por ejemplo, que $x$ 'está fundado en' $y$ consiste en el hecho de que $\square((y$ existe $) \rightarrow$ (x existe $))$. Sucede, sin embargo, que esto hace que todo funde ontológicamente a entidades necesarias. Si, por ejemplo, el número 3 es una entidad necesaria, entonces todo es fundamento del número 3. Tal vez uno rechace la existencia de números u otras entidades matemáticas como entidades necesarias. Podría, sin embargo, aceptarse la existencia de estados de cosas necesarios por su simple forma lógica, tal como el estado de cosas de 'haber unicornios o no haber unicornios'. Todo sería fundante ontológicamente de haber unicornios o no haber unicornios, lo que choca contra nuestras intuiciones ontológicas.

Si no parece que la noción de 'fundación' pueda ser analizada mediante recursos modales - $\mathrm{u}$ otros- entonces lo más razonable es pensar que se trata de una noción ontológica primitiva, no analizable por otras. Es más, probablemente las mismas nociones modales puedan ser iluminadas por la relación de fundación más bien que al revés (cf. Rosen, 2010; Correia, 2012; Koslicki, 2012). Como sucede en tantos otros casos, sin embargo, el hecho de que la noción de 'fundación' sea primitiva no implica que sea, en algún sentido, oscura o que permita sostener cualquier cosa acerca de qué funda qué ontológicamente. Se puede dilucidar la noción de 'fundación' mediante la indicación del tipo de conexiones teorías que posee esta noción con otras y las restricciones que debe satisfacer. Esto es lo que se ha hecho en la discusión más reciente (cf. Correia y Schnieder, 2012b). No es raro que, examinado el concepto con más detalle, resulte que no hay un único concepto, sino una familia de ellos. Aquí se va a utilizar un concepto

\footnotetext{
${ }^{4}$ Estos problemas no obstan a que, tal como se indicará más adelante, de una relación de fundación auténtica entre $x$ e $y$ se deriva un bi-condicional estricto. La cuestión es que no vale la conversa. Un bi-condicional estricto es más débil que una relación de fundación.
} 
de ‘fundación' que parece el más razonable para considerar las cuestiones sistemáticas que surgen a propósito de la relación que debe tener un estado de cosas con sus componentes, pero, cuando esto sea relevante, se hará también indicación de las alternativas abiertas.

Una primera cuestión es si la relación de fundación debe tomarse como un predicado o como un operador sentencial. Tomado del primer modo, la relación de fundación se expresa por el predicado "está fundado en" que debe ser flanqueado por nombres u otras expresiones denotativas de entidades del tipo apropiado -estados de cosas, objetos, universales, etcétera. Tomado del segundo modo, los enunciados de fundación deben hacerse mediante un operador o conectivo que vincula entre sí oraciones. Este conectivo puede ser, por ejemplo, el operador porque entre oraciones. Por un lado, la perspectiva predicativa parece menos económica, pues requiere precisar el tipo de categorías ontológicas que pueden estar siendo conectadas por relaciones de fundación, mientras que la perspectiva conectiva no parece tener estos requerimientos. Desde otras perspectivas, la perspectiva conectiva parece traer consigo dificultades innecesarias. Por ejemplo, considérese cómo podría ser analizado el concepto de 'fundamental' (cf. Correia y Schnieder, 2012b, 11). Una forma muy natural de análisis desde la perspectiva predicativa es la siguiente:

(2) $x$ es fundamental $={ }_{\mathrm{df}} \forall y \neg(x$ está fundado en $y)$

Desde una perspectiva conectiva, en cambio, debería analizarse como:

(3) Es fundamental que $p={ }_{\mathrm{df}} \forall q \neg(p$ porque q)

Las variables ' $p$ ' y ' $q$ ' tienen como rango oraciones de algún lenguaje. Lo que enuncia una oración " $p$ ” es fundamental, de acuerdo al análisis (3) si y sólo si no hay una oración " $q$ " perteneciente al lenguaje de que se trate, tal que resulte verdadero enunciar que " $p$ porque $q$ ". Es obvio que, entonces, lo que resulta enunciado por una oración de un lenguaje podría resultar fundamental debido a la pobreza de recursos expresivos del lenguaje de que se trate. Un lenguaje con pocos recursos conceptuales puede generar hechos 
'fundamentales' curiosos. ${ }^{5}$ Se supondrá, por lo tanto, en lo que sigue que la relación de fundación queda correctamente expresada por un predicado multígrado de este tipo: $x$ está fundado en $y_{1}, y_{2}, \ldots, y_{\mathrm{n}}$. Lo que se encuentra designado por la o las expresiones a la derecha se designarán como "el fundamento" y lo que se encuentra designado a la izquierda se designará como "lo fundado". Lo normal ha sido suponer que los relata de la relación de fundación son 'hechos'. Probablemente, esto permite simplificar la discusión general de los rasgos sistemáticos de la relación de fundación. Para lo que interesa aquí, sin embargo, interesa una relación de fundación no sólo multígrada, sino además apta para conectar entre sí entidades de diferentes categorías ontológicas como lo son estados de cosas o hechos, objetos, universales y-eventualmente- tiempos.

Hay una serie de intuiciones que la relación de fundación parece satisfacer. La relación de fundación parece ser reflexiva, asimétrica e irreflexiva. Esto es, parece intuitivo que nada está fundado en sí mismo; que si $x$ está fundado en $y$, entonces $y$ no está fundado en $x$, que si $x$ está fundado en $y$ e $y$ está fundado en $z$, entonces $x$ está fundado en $\%$ De la existencia de una relación de fundación entre ciertas entidades parece seguirse, también, un patrón de covarianza modal entre esas entidades. Se podría, entonces, sostener que la relación de fundación satisface los siguientes postulados: ${ }^{6}$

$$
\square \forall x \forall y \square((x \text { está fundado en } y) \rightarrow \square((x \text { existe }) \leftrightarrow(y \text { existe })))
$$

$$
\square \forall x \square \neg(x \text { está fundado en } x)
$$

\footnotetext{
5 Supóngase, en efecto, un lenguaje L con sólo dos predicados: "es un perro" y "es un gato". En este lenguaje, con el recurso de cuantificadores, pueden formarse las oraciones "hay un perro" y "hay un gato". La oración "hay un perro porque hay un gato" es falsa. Entonces, sería fundamental que hay perros, pues no hay ninguna oración $q$ en $\mathrm{L}$ con la que pueda formarse una oración verdadera de la forma "hay un perro porque $q$ ". Esto no muestra, sin embargo, ningún carácter ontológicamente 'profundo' acerca de la existencia de perros, sino simplemente las carencias expresivas de L.

${ }^{6}$ Puede ser útil confrontar estos postulados con la formulación de lo que Fine llama la "lógica pura de la fundación" mediante reglas estructurales. Cf. Fine, 2012b, 54-57; 2012a. Las reglas de Fine incluyen también las nociones de 'fundación parcial' y de 'fundación débil' de que se tratará más abajo.
} 
(6) $\square \forall x \forall y \square((x$ está fundado en $y) \rightarrow \neg(y$ está fundado en $x))$

(7) $\square \forall x \forall y \forall z \square(((x$ está fundado en $y) \wedge(y$ está fundado en $y)) \rightarrow(x$ está fundado en $\approx$ ))

La tesis (4) enuncia que la existencia de una relación de fundación entre $x$ e $y$ implica que tales entidades son modalmente correlativas. ${ }^{7}$ Por supuesto, no vale la conversa. En todo mundo posible en que exista $x$ debe también existir $y$, y en todo mundo posible en que exista $y$ debe también existir $x$. Cuando una entidad está fundada en otra, la mera existencia del fundamento es suficiente para la existencia de la entidad fundada. La entidad fundada, sin embargo, no podría existir sino por la existencia de su fundamento. El fundamento es, también, necesario para la existencia de lo fundado.

La noción de dependencia ontológica parece poder ser analizada en términos de la relación de fundación. Usualmente se ha sostenido que una entidad $x$ depende ontológicamente de $y$ si es que $x$ no podría existir si es que $y$ no existiese. Tal como se ha explicado arriba para la relación de fundación, no sería apropiado intentar analizar la relación de dependencia ontológica por una implicación estricta. Un condicional estricto del tipo $\square((x$ existe $) \rightarrow(y$ existe $)$ ) puede ser verdadero de dos ítems $x$ e $y$, sin que sea verdadero en virtud de lo que $x$ e $y$ son, sino, por ejemplo, por el hecho de que $y$ es una entidad abstracta necesaria, aunque no tenga ninguna conexión

\footnotetext{
${ }^{7}$ Este es un punto en donde hay diferencias terminológicas. Aunque consenso en que la existencia de relaciones de fundación trae consigo consecuencias modales, algunos llamarían el tipo de fundación caracterizada aquí por (4) como 'fundación total'. La relación de fundación, para ellos, podría ser más débil y estar caracterizada por:

$\left(4^{\prime}\right) \square((x$ está fundado en $y) \rightarrow \square((y$ existe $) \rightarrow(x$ existe $)))$

O bien por:

$\left(4^{\prime \prime}\right) \square((x$ está fundado en $y) \rightarrow \square((x$ existe $) \rightarrow(y$ existe $)))$

La formulación $\left(4^{\prime \prime}\right)$ tiende a identificar la fundación con la dependencia ontológica. No hay nada malo de por sí con estas otras nociones de fundación, en la medida en que las nociones caracterizadas sean precisas. Interesa, sin embargo, para los objetivos de este trabajo centrados en las conexiones entre un estado de cosas y sus componentes y el regreso de Bradley concentrar la atención en la noción de fundación más fuerte que satisface (4).
} 
con $x$. De todos modos, se puede suponer que la relación de dependencia ontológica satisface:

(8) $\square \forall x \forall y \square((x$ depende de $y) \rightarrow \square((x$ existe $) \rightarrow(y$ existe $)))$

La dependencia ontológica de $x$ en $y$ se sigue de la fundación de $x$ en $y$. Se trata de una relación más débil que la de fundación, pues $x$ podría depender de $y$ aunque $x$ no esté fundado en $y$. Esto acaecería si es que en todo mundo posible en que $x$ exista $y$ también exista, pero no en todo mundo posible en que $y$ exista, $x$ también exista. En otras palabras, se daría una relación de dependencia ontológica sin fundación entre $x$ e $y$, si es que la existencia de $y$ fuese necesaria para la existencia de $x$, pero no fuese suficiente para la existencia de $x$. La relación de dependencia ontológica parece corresponder a la noción de 'fundación parcial' (cf. Fine, 2012b, 50). De un modo general se puede sostener que:

(9) ( $x$ está fundado parcialmente en $y)={ }_{\mathrm{df}} \exists z_{1} \ldots z_{\mathrm{n}}(x$ está fundado en $y$, $\left.z_{1}, \ldots, z_{n}\right)$

Como no es necesario que $z_{i} \neq y$, se sigue que si $x$ está fundado en $y$, entonces $x$ está fundado parcialmente en $y$. No vale la conversa, naturalmente. Un caso en que $x$ esté fundado parcialmente en $y$, pero en que $x$ no esté fundado en $y$ sería un caso en que la existencia de $y$ sería necesaria para la existencia de $x$, pero no sería suficiente para la existencia de $x$. Por este motivo, la noción de fundación parcial parece poder identificarse con la noción de dependencia ontológica. Hay prevenciones para esta identificación, sin embargo, que se indicarán más adelante.

Así como se posee un concepto de fundación que es irreflexivo y asimétrico, tal como aparece en (5) y (6) se puede especificar un concepto de 'fundación débil' de un modo análogo a como en mereología se posee el concepto de 'parte impropia' junto con el concepto de 'parte propia'. La idea fundamental es:

(10) $(x$ está fundado débilmente en $y)=_{\mathrm{df}}((x=y) \vee(x$ está fundado en $y))$

Pero también uno podría utilizar como noción primitiva la de fundación débil y analizar mediante ella la de fundación 'estricta': 
(11) ( $x$ está fundado estrictamente en $y)=_{\mathrm{df}}((x \neq y) \wedge(x$ está fundado débilmente en $y$ )

Se entenderá aquí la relación de 'fundación' simplemente como la fundación 'estricta'. La combinación de la distinción entre las nociones de fundación estricta y fundación débil, y entre las nociones de fundación parcial y fundación total genera una serie de alternativas no equivalentes entre sí: estricta total, estricta parcial, débil total y débil parcial (cf. Fine, 2012b, 53-54). Estas diferentes nociones no serán consideradas aquí. La introducción de las nociones de fundación débil y fundación parcial permiten agregar a los postulados (4)-(7) los siguientes:

$\square \forall x \square$ ( $x$ está fundado débilmente en $x$ )

$\square \forall x \forall y \square(((x$ está fundado débilmente en $y) \wedge(y$ está fundado débilmente en $x)) \rightarrow(x=y))$

(14) $\square \forall x \forall y \square((x$ está fundado en $y) \rightarrow(x$ está fundado débilmente en $y))$

Considerando estos conceptos y estas relaciones sistemáticas, interesará ahora examinar el modo en que un estado de cosas está fundado en sus componentes.

\section{2. ¿Está fundado un estado de cosas en sus constituyentes?}

Los hechos acerca de la fundación de ciertas entidades en otras no son reducibles a la distribución de hechos modales. Hechos modalmente conectados pueden no estar fundados entre sí. La existencia de la suma mereológica $(x+y)$ está correlacionada modalmente a la existencia de $x$ y a la existencia de $y$. Esto es, en todo mundo posible en que exista $(x+y)$ existe también $x$ y existe también $y$, y en todo mundo posible en que exista $x$ y exista $y$, existe también $(x+y)$. La existencia de $x$ y de $y$ no está fundada en la existencia de $(x+y)$, sino al revés. Será útil, sin embargo, el hecho de que la existencia de relaciones de fundación implica la existencia de correlaciones modales (cf. Correia y Schnieder, 2012b, 20-22; Correia, 2012; Koslicki, 2012). El tipo de fundación que interesaría aquí debería darse apelando expresamente al carácter multígrado de tal relación y suponiendo 
que el fundamento es una pluralidad multi-categorial de universales, objetos y tiempos. Esto es, para un estado de cosas $\left[U x_{1} x_{2} \ldots x_{n} \nexists\right.$ debería valer que:

(15) $\left[U x_{1} x_{2} \ldots x_{n} \nexists\right.$ está fundado en $U, x_{1}, x_{2}, \ldots, x_{n}, t$

Por instanciación universal en el principio modal (4) se sigue que:

(16) $\square\left(\left(\left[U x_{1} x_{2} \ldots x_{n} \nexists\right]\right.\right.$ está fundado en $\left.U, x_{1}, x_{2}, \ldots, x_{n}, t\right) \rightarrow \square\left(\left(\left[U x_{1} x_{2} \ldots\right.\right.\right.$ $x_{\mathrm{n}} t$ existe $) \leftrightarrow\left((U\right.$ existe $) \wedge\left(x_{1}\right.$ existe $) \wedge\left(x_{2}\right.$ existe $) \wedge \ldots \wedge\left(x_{n}\right.$ existe $) \wedge(t$ existe))))

Por modus ponens sobre (15) y (16) se sigue ahora que:

(17) $\square\left(\left(\left[U x_{1} x_{2} \ldots x_{n} \nexists\right.\right.\right.$ existe $) \leftrightarrow\left((U\right.$ existe $) \wedge\left(x_{1}\right.$ existe $) \wedge\left(x_{2}\right.$ existe $) \wedge \ldots$ $\wedge\left(x_{\mathrm{n}}\right.$ existe $) \wedge(t$ existe $\left.)\right)$

Pero (17) parece falso. Es posible que existan todos los constituyentes del estado de cosas $\left[U x_{1} x_{2} \ldots x_{n}\right]$ y que, sin embargo, éste no exista. Los objetos $x_{1}, x_{2}, \ldots, x_{n}$ podrían no estar instanciando $U$, sino otro universal $U^{\prime}$. Si el universal $U$ fuese un universal trascendente, existirá por igual sea que tenga instancias o no. Supóngase, sin embargo, que se trata de un universal inmanente que sólo existe en un mundo posible si es que tiene instancias en ese mundo. En este caso, también podría existir el universal $U$ aunque no el estado de cosas $\left[U x_{1} x_{2} \ldots x_{n}\right]$, si es que -por ejemplo- estuviese instanciado en objetos diferentes de $x_{1}, x_{2}, \ldots, x_{n}$. Por último, podría suceder que $U$ estuviese efectivamente instanciado en $x_{1}, x_{2}, \ldots, x_{n}$, pero no en el instante de tiempo $t$, sino en uno diferente, sea $t^{\prime}$. De este modo, no vale el bicondicional estricto (17) de derecha a izquierda. Por modus tollens sobre (16) se sigue, entonces, que (15) es falso. El estado de cosas $\left[U x_{1} x_{2} \ldots\right.$ $x_{n} t$ no está fundado en $U, x_{1}, x_{2}, \ldots, x_{n}, t$.

No hay ningún problema, sin embargo, en sostener la dependencia o fundación parcial de un estado de cosas respecto de sus componentes. Ciertamente, la existencia de un estado de cosas $\left[U x_{1} x_{2} \ldots x_{n} t\right]$ exige como condición necesaria la existencia de $U, x_{1}, x_{2}, \ldots, x_{\mathrm{n}}, t$. Resulta verdadero el condicional estricto: 
(18)

$\square\left(\left(\left[U x_{1} x_{2} \ldots x_{n}\right]\right.\right.$ existe $) \rightarrow\left((U\right.$ existe $) \wedge\left(x_{1}\right.$ existe $) \wedge\left(x_{2}\right.$ existe $) \wedge \ldots$ $\wedge\left(x_{\mathrm{n}}\right.$ existe $) \wedge(t$ existe $\left.)\right)$

Tal como se ha indicado la verdad de un condicional estricto no es suficiente para sostener la existencia de una auténtica dependencia ontológica. Sin embargo, como la identidad de los componentes de un estado de cosas - universal, objetos y tiempo- vienen a integrar las condiciones de identidad del estado de cosas (cf. Fine, 1995; Lowe, 2006, 198-201), no hay duda de que aquí estamos en presencia de una auténtica dependencia ontológica.

El hecho de que un estado de cosas no esté fundado en sus constituyentes permite comprender de un modo nuevo el famoso regreso de Bradley (cf. 1897, 17-18). Tal como usualmente es comprendido, el regreso surge porque se requiere dar una 'explicación' de la diferencia entre una fusión mereológica de los componentes de un estado de cosas, o un conjunto cuyos elementos son exactamente los componentes de un estado de cosas, o, en fin, una pluralidad constituida por los componentes de un estado de cosas y el estado de cosas propiamente tal. La explicación vendría dada porque, si se trata -por ejemplo- del estado de cosas $\left[U x_{1} x_{2} \ldots x_{n}\right]$, el universal $U$ está realmente instanciado en $x_{1}, x_{2}, \ldots, x_{\mathrm{n}}$ en el instante $t$, mientras que esto no sucede en una fusión mereológica, o un conjunto, o una pluralidad de esos mismos constituyentes. ¿Qué es lo que debería ser, sin embargo, la 'instanciación' de un universal en ciertos particulares? Parece tratarse de una relación. Un filósofo que sostenga de manera general que las relaciones son universales debe, por lo tanto, sostener también que la relación de 'instanciación' es un universal tal como cualquier otro. La 'explicación' que se estaría dando acerca de por qué hay una diferencia entre el estado de cosas $\left[U x_{1} x_{2} \ldots x_{n}\right]$ y una suma, conjunto o pluralidad constituida por $U, x_{1}, x_{2}, \ldots, x_{n}, t$, es que en el estado de cosas interviene un universal relacional de 'instanciación', sea $I$, que se encuentra conectando entre sí los componentes del estado de cosas $\left[U x_{1} x_{2} \ldots x_{n} \sharp\right.$.

La relación de instanciación $I$ a que se hace mención aquí tiene varias peculiaridades. Debe tratarse de una relación de orden superior, pues no sólo debe encontrarse instanciada en objetos -y tiempos- sino que también en otros universales de primer orden -que se instancian en objetos. Como estos universales pueden tener cualquier adicidad, la relación de instancia- 
ción $I$ debe ser multígrada. Uno puede suponer que la relación de instanciación posee un tipo lógico preciso, por lo cual la instanciación de la relación de instanciación de tipo $n$ debería ser una relación diferente de tipo $n+1$. Bajo la suposición de que hay relaciones de instanciación de tipos lógicos determinados surge una infinidad de tales relaciones, una para cada tipo lógico. Uno también, sin embargo, podría suponer que sólo hay una única relación de instanciación sin tipo lógico determinado y que puede, por lo tanto, estar instanciada en sí misma. Estas peculiaridades y alternativas no son relevantes para lo que sigue. El problema que genera la postulación de una relación de instanciación $I$ como explicación de la diferencia de un estado de cosas auténtico y una mera suma, conjunto o pluralidad es que, al tratarse tal relación de un universal como cualquier otro, se requiere que se encuentre efectivamente instanciado. Esto es, se supone que hay un estado de cosas que se encuentra constituido por los mismos elementos de $\left[U x_{1} x_{2} \ldots x_{n}\right]$, pero, además, por la relación de instanciación $I$, sea $\left[I U x_{1} x_{2}\right.$ $\ldots x_{n} A$, pero este estado de cosas nuevamente no se encuentra fundado en sus constituyentes $I, U, x_{1}, x_{2}, \ldots, x_{\mathrm{n}}, t$. Los motivos son los mismos que los indicados arriba. Si el estado de cosas $\left[I U x_{1} x_{2} \ldots x_{n} t\right]$ estuviese fundado en sus componentes, entonces, por el principio modal (8) debería resultar que:

$$
\square\left(\left([ I U x _ { 1 } x _ { 2 } \ldots x _ { n } \nexists \text { existe } ) \leftrightarrow \left(( I \text { existe } ) \wedge ( U \text { existe } ) \wedge ( x _ { 1 } \text { existe } ) \wedge \left(x_{2}\right.\right.\right.\right.
$$
existe $) \wedge \ldots \wedge\left(x_{n}\right.$ existe $) \wedge(t$ existe $\left.\left.)\right)\right)$

Pero este bicondicional estricto parece falso de derecha a izquierda, pues podrían existir todos los constituyentes de $\left[\begin{array}{llll}I U x_{1} x_{2} & \ldots x_{n} t\end{array}\right]$, sin embargo, no estar efectivamente instanciada la relación $I$ en $U, x_{1}, x_{2}, \ldots$, $x_{\mathrm{n}}$ en el instante $t$. Entonces, por modus tollens, se sigue que $\left[I U x_{1} x_{2} \ldots x_{n} t\right]$ no está fundado en $I, U, x_{1}, x_{2}, \ldots, x_{\mathrm{n}}, t$. La relación de instanciación podría existir -suponiendo que se trata de un universal inmanente- si es que estuviese instanciada en otros universales y objetos diferentes de $U, x_{1}, x_{2}, \ldots$, $x_{n}$. El universal $U$ podría existir, tal como se explicó arriba, si es que estuviese instanciado en otros objetos diferentes de $x_{1}, x_{2}, \ldots, x_{n}$. Etcétera. Es obvio que si uno ahora pretendiese resolver este problema apelando nuevamente al hecho de que la relación de instanciación $I$ se encuentra realmente instanciada en $U, x_{1}, x_{2}, \ldots, x_{\mathrm{n}}, t$, no se va a avanzar nada. Se trataría del hecho de que la relación de instanciación -sea la misma relación $I$ o una 
relación de un tipo lógico superior- está instanciada en $I, U, x_{1}, x_{2}, \ldots, x_{n}$, t. Pero la relación de instanciación a la que se apele -sea la misma u otra de tipo superior- es simplemente un universal como cualquier otro que podría existir sin estar instanciado de hecho en $I, U, x_{1}, x_{2}, \ldots, x_{n}, t$. Por los mismos motivos ya indicados, el estado de cosas $\left[I^{2} I^{1} U x_{1} x_{2} \ldots x_{n}\right]^{8}$ no está fundado en $I^{2}, I^{1}, U, x_{1}, x_{2}, \ldots, x_{\mathrm{n}}, t$.

El famoso regreso de Bradley se genera aquí porque cualquier otra ocurrencia de la relación de instanciación, o de relaciones de instanciación de tipos lógicos más altos tendrán siempre el mismo problema. En ningún nivel se encontrará una base de entidades que puedan fundar el estado de cosas de que se trate. A veces se ha presentado este problema como un problema acerca de cómo "analizar" un estado de cosas (cf. Armstrong, 1978a, 18-21, 41-42, 53-56, 69-71, 106-107) o acerca de cómo "explicar ontológicamente" un estado de cosas. Ambas nociones aplicadas aquí oscurecen innecesariamente la naturaleza del problema. La cuestión acerca del 'análisis' de un concepto tiene que ver con lo que pueda atribuirse de manera necesaria a todo aquello que satisfaga el analysandum mediante una reflexión a priori acerca de él. No es nada claro que aquí lo que interese sea el concepto de 'estado de cosas'. Más bien, lo que interesa es comprender qué es un estado de cosas y qué relación tiene un estado de cosas con sus componentes. Se ha utilizado, por otra parte, el concepto de 'explicación' para indicar cómo es que los antecedentes causales de un evento permiten aquietar nuestras expectativas epistémicas acerca de por qué acaece ese evento. No se está buscando algo de este tipo cuando se busca indicar la diferencia entre un estado de cosas y una mera fusión mereológica, clase o pluralidad. No es necesario tratar las cuestiones propiamente ontológicas como cuestiones acerca del carácter de nuestros conceptos, o como cuestiones acerca de conexiones causales informativas. Si se quiere hablar, en cambio, de una 'explicación no-causal', esto es exactamente lo que es una relación de fundación.

\footnotetext{
${ }^{8}$ Sea aquí $I^{1}$ la primera ocurrencia de la relación de instanciación $I$, o bien la relación de instanciación $I$ de un tipo lógico determinado $n$. Sea $I^{2}$ la segunda ocurrencia de la relación de instanciación -instanciada en sí misma- o bien la relación de instanciación $I$ de un tipo lógico $n+1$, superior al tipo lógico que tuviese $I^{1}$.
} 


\section{Lecciones del regreso de Bradley}

El regreso de Bradley ha sido tomado como una razón para adoptar diferentes posiciones en ontología. Algunos han sostenido que la instanciación no debe ser considerada como una relación tal como cualquier otra, sino como un hecho ontológico primitivo (cf. Strawson, 1959, 167-168; Bergmann, 1967, 9-12; Armstrong, 1978a, 108-113; 1989, 108-110; 1997, 127; 2004, 46-48). Otros han sostenido que el mismo universal -sea relacional o no- es aquello que efectúa por sí mismo la unidad del estado de cosas (cf. Grossmann, 1973, 143-144; 1983, 8; Chisholm, 1996, 54). Otros han sostenido que los estados de cosas son entidades que no pueden ser 'reducidas', 'analizadas' o 'explicadas' por otras entidades (cf. Hochberg, 1978, 338-339). Otros han sostenido que el regreso de Bradley no es realmente vicioso, pues una secuencia infinita de aplicaciones recursivas de la relación de instanciación $I$, o bien una secuencia de infinitas relaciones de instanciación diferentes, de diferentes tipos lógicos, permite efectuar un 'análisis' o 'explicación' adecuado (cf. Gaskin, 2008, 354-355; Orilia, 2009). Algunos han sostenido, en fin, que los estados de cosas requieren de algo que funcione como unificador 'externo' del estado de cosas (cf. Vallicella, 2000, 2002).

La formulación en términos de relaciones de fundación permite iluminar todas estas diferentes alternativas. Interesa ahora, sin embargo, considerar una en particular en donde se salva la fundación de los estados de cosas en sus componentes. Una relación universal de instanciación no permite suplementar una fundación para un estado de cosas -ofrece algo de lo que el estado de cosas parece depender, naturalmente, pero no una fundación. La situación varía radicalmente, sin embargo, si es que se postula una relación de carácter particular y que, esencialmente, deba ser la instanciación del universal que compone un estado de cosas en los objetos que componen ese mismo estado de cosas, y en el tiempo preciso en que lo componen. Una relación de carácter particular es un tropo. Un tropo, entonces, de las características indicadas, permite restablecer la fundación del estado de cosas en sus componentes y, con ello, permite evitar el regreso de Bradley. Esto no implica eliminar los universales por clases de semejanza de tropos, ni tampoco implica eliminar los objetos particulares por cúmulos de tropos co-instanciados entre sí. Por supuesto, tampoco es incompatible con tales ideas. La cuestión es simplemente que, si uno defiende una ontología de estados de cosas con universales y objetos particulares, y si uno 
adicionalmente está inclinado a pensar que los estados de cosas deben estar fundados en sus componentes, entonces hay buenos motivos para postular que junto a los universales, objetos particulares y tiempos, debe haber también tropos que sean esencialmente la instanciación de esos universales en esos objetos en esos tiempos. Intuiciones en este mismo sentido ya han sido propuestas con anterioridad (cf. Mertz, 1996, 184-195; Meinertsen, 2008; Wieland y Betti, 2008; Maurin, 2011) pero sin introducir relaciones de fundación que hacen la situación teórica mucho más perspicua. Para el caso de un estado de cosas $\left[U x_{1} x_{2} \ldots x_{n} \rrbracket\right]$ supóngase un tropo $U$-en- $x_{1}, x_{2}, \ldots, x n$, $t$. Tal como se ha indicado, debe suponerse que es parte de las condiciones de identidad de tal tropo el ser precisamente la instanciación de $U$ y no de otro universal, y el estar instanciado en $x_{1}, x_{2}, \ldots, x_{n}, t$ y no otros objetos y otro instante de tiempo. Entonces:

(20) $\left[U x_{1} x_{2} \ldots x_{n} t\right.$ está fundado en $U, x_{1}, x_{2}, \ldots, x_{n}, t, U$-en- $x_{1}, x_{2}, \ldots, x_{n}, t$

Si se hace ahora el test por el que la fundación de los estados de cosas en sus componentes resultó desechada, se verá que no hay ningún problema con el bicondicional estricto que debería estar implicado por (20), de acuerdo al principio modal (4). En efecto, si el estado de cosas $\left[U x_{1} x_{2} \ldots\right.$ $x_{n} t$ está fundado en sus constituyentes, y éstos incluyen el tropo $U$-en- $x_{1}$, $x_{2}, \ldots, x_{\mathrm{n}}, t$, entonces, se seguiría que:

(21) $\square\left(\left(\left[U x_{1} x_{2} \ldots x_{n} t\right]\right.\right.$ existe $) \leftrightarrow\left((U\right.$ existe $) \wedge\left(x_{1}\right.$ existe $) \wedge\left(x_{2}\right.$ existe $) \wedge \ldots$ $\wedge\left(x_{n}\right.$ existe $) \wedge(t$ existe $) \wedge\left(U\right.$-en- $x_{1}, x_{2}, \ldots, x_{n}, t$ existe $\left.)\right)$

Este bicondicional estricto es verdadero de izquierda a derecha pues hay dependencia ontológica del estado de cosas respecto de sus componentes, tal como lo había antes. Lo interesante es que el bicondicional estricto (21) también es verdadero de derecha a izquierda, pues en todo mundo posible en que existan el universal $U$, los objetos $x_{1}, x_{2}, \ldots, x_{n}$, el tiempo $t$ y el tropo $U$-en- $x_{1}, x_{2}, \ldots, x_{n}, t$ tiene que existir el estado de cosas $\left[U x_{1} x_{2} \ldots x_{n} t\right]$. Dado que es esencial para el tropo $U$-en- $x_{1}, x_{2}, \ldots, x_{n}, t$ ser la instanciación de $U$ en esos objetos en ese tiempo, no podría existir el universal $U$ sin estar instanciado por tales objetos en ese tiempo, si es que existe el tropo en cuestión. Fácilmente se puede ver cómo aquí la postulación de un tropo con las características indicadas evita que se dispare el 
regreso de Bradley, pues no se requiere nada adicionalmente a los componentes del estado de cosas para fundar su unidad como tal.

Para que un tropo pueda cumplir estas funciones en la fundación de los estados de cosas se requiere que tenga un perfil modal muy definido. Debe ser parte de las condiciones de identidad de un tropo en qué objeto u objetos se encuentre instanciado, cuándo se encuentre instanciado y de qué universal sea la instanciación. Usualmente, los defensores de tropos pretenden eliminar o reducir los universales a clases de tropos perfectamente semejantes entre sí o clases naturales de tropos, por lo que la conexión del tropo con un universal no es algo que sea objeto usual de consideración. Cuando se trata, sin embargo, de la conexión de un tropo con el objeto particular en que se 'encuentra' o que lo 'posee', hay una enorme variedad de posiciones. Algunos han sostenido que en un objeto particular junto con los tropos debe postularse un sustrato, 'particular desnudo' o 'particular delgado' que los instancie (cf. Martin, 1980; Heil, 2003, 137-150, 169-178; 2012, 12-52). Estos filósofos están inclinados a pensar que es parte de las condiciones de identidad de un tropo el estar instanciado en un sustrato específico. Por lo tanto, en estas concepciones un tropo no puede 'transmigrar' de un objeto a otro. La mayoría de los defensores de ontologías de tropos, sin embargo, han pretendido no sólo reducir los universales a clases de tropos, sino que también los objetos particulares (cf. Williams, 1953a, 1953b; Campbell, 1990; Maurin, 2002, 117-180; Ehring, 2011, 98-135). La motivación central para las ontologías de tropos ha sido conseguir una teoría uni-categorial. Por supuesto, lo que se está presentando aquí es una motivación diferente, que tiene que ver con requerimientos sistemáticos internos de las ontologías de universales. Cuando se consideran las cosas desde la perspectiva de una teoría general de tropos, donde todo lo que hay en la realidad son entidades de tal categoría, no parece de entrada necesario atribuir a los tropos un perfil modal muy preciso. En las defensas 'clásicas' de ontologías uni-categoriales de tropos se ha adoptado una posición máximamente liberal respecto a la combinatoriedad de un tropo con otros. Los tropos pueden estar libremente juntos o separados entre sí. Un tropo puede estar integrando o no un cúmulo junto con otros tropos, y puede estar integrando un cúmulo en un instante de tiempo, y no estarlo integrando en otro instante de tiempo posterior. Un tropo conforma un cúmulo -y, con ello, un objeto particular- simplemente por el hecho de estar co-ocupando una región del espacio en un tiempo o, si se prefiere, por estar co-ocupando con 
otros tropos una región del espacio-tiempo. La conformación de cúmulos de tropos es un hecho que no debe ser más misterioso que la ocupación de una región del espacio. No hay aquí ningún tipo de conexión modal más profunda entre un tropo y otros.

Una teoría de este tipo se aviene bien a inclinaciones humeanas, pues no hay mayores restricciones para que cualquier tropo exista junto o separado de cualquier otro. La 'co-ocupación' de la misma región del espacio, sin embargo, es menos inocente de lo que parece a primera vista. Si se tratase de un tropo, genera un regreso semejante al regreso de Bradley. Si no se trata de un tropo, sino de un hecho ontológicamente primitivo, la economía general de la teoría comienza a perderse. ${ }^{9}$ Por estos motivos otras concepciones han postulado un perfil modal exigente para los tropos que van a conformar un cúmulo (cf. Simons, 1994). Se ha sostenido, por ejemplo, que los tropos que conforman un cúmulo deben ser todos ellos dependientes ontológicamente entre sí, de manera que ninguno de esos tropos podría existir sin que existiesen todos los restantes tropos que conforman ese cúmulo. En una teoría de este tipo no se hace necesario postular hechos primitivos de co-presencia o de otro tipo para evitar un regreso semejante al regreso de Bradley. Otras teorías, en este mismo espíritu, diferencian entre

${ }^{9}$ En efecto, la relación de co-ocupación que conecta a diferentes tropos entre sí difiere en respectos importantes de la relación de ocupación entre un objeto y una región del espacio. La co-ocupación entre tropos debe poder excluir ciertos tropos, pero no otros. Los tropos determinados bajo la misma propiedad determinable se excluyen entre sí. Si en una región del espacio hay un tropo de masa de k gramos, entonces están automáticamente excluidos tropos de masa de $k+n$ gramos en esa misma región. No cualquier tropo puede estar, entonces, junto con cualquier otro. Uno podría estar inclinado, también, a pensar que cualquier región del espacio debería seleccionar un y sólo un cúmulo de tropos: exactamente el cúmulo de todos los tropos que están co-ocupando tal región. Esto dejaría espacio para cúmulos con tropos incompatibles entre sí. En una concepción de este estilo, por otro lado, por definición no puede haber objetos diferentes en la misma región del espacio. Es una verdad empírica, sin embargo, que hay tales objetos. Acomodar estos hechos empíricos en la teoría obligaría a discriminar entre relaciones de co-ocupación que sí dan lugar a un cúmulo de las que no lo hacen. La relación de 'co-ocupación' a la que debería hacer apelación, por lo tanto, un defensor de una ontología de tropos, difiere sustantivamente de nuestras intuiciones ordinarias acerca de la 'ocupación' del espacio por un objeto -nuestro concepto ordinario- y no es nada inocente. 
un 'núcleo' y una 'periferia' en el cúmulo de tropos. Los tropos del núcleo están todos ellos dependientes entre sí, por lo que ninguno de ellos podría existir sin que existiesen todos los restantes. Los tropos de la periferia, en cambio, podrían no existir y ello no impediría que existiesen los tropos del núcleo, aunque estos tropos periféricos no pueden existir sino junto a los tropos del núcleo. Los tropos periféricos son, entonces, dependientes de los tropos del núcleo, pero no al revés.

La concepción de los tropos que se aviene a lo que quiere proponerse aquí debe ser alguna de las que postula un perfil modal exigente para estos. Es neutral en cuanto a si los tropos deben estar o no instanciados en un sustrato. Es neutral en cuanto a si los tropos deben estar conformando un cúmulo, ya sea porque todos los tropos que conforman tal cúmulo son dependientes entre sí, o ya sea porque hay un núcleo y una periferia en ese cúmulo. Se requiere, en cualquier caso, que sea esencial para el tropo conformar el objeto que de hecho conforma, comoquiera sea concebida la naturaleza de tal 'objeto'. Algo análogo sucede con el universal y el tiempo que conforman el estado de cosas. Si se quiere, la concepción que se está presentando resulta más cercana de la ontología de 'cuatro categorías' de Jonathan Lowe (cf. Lowe, 2006, 20-51). Lowe es un defensor de una teoría relativamente tradicional de universales y particulares. Lo que resulta peculiar en su concepción, sin embargo, es que junto a las propiedades universales postula 'modos' que son la instanciación de esas propiedades universales en el objeto particular. ${ }^{10}$

Se puede apreciar aquí que no se requiere sostener que la 'instanciación' es una relación peculiar o un tipo de relación peculiar -si es que uno va a introducir diferentes relaciones para diferentes tipos lógicos. No hay una única relación de instanciación ni siquiera para un tipo lógico determinado. Tampoco se requiere sostener que la instanciación designa un 'vínculo

\footnotetext{
${ }^{10}$ Se distingue también entre propiedades universales - cuyas instanciaciones son 'modos'- y kinds o universales sustanciales cuyas instanciaciones son sustancias particulares. Las cuatro categorías fundamentales son, entonces: propiedades, universales sustanciales, modos y sustancias particulares. Los universales sustanciales están 'caracterizados' por propiedades. Las sustancias particulares, a su vez, están también ‘caracterizadas' por los modos. La ‘instanciación’ es una relación que sólo se da entre universales y particulares, pero no entre universales o entre particulares. Para este caso, la relación es la 'caracterización'.
} 
no-relacional' (non-relational tie) o un 'vínculo más estrecho que una relación' o un hecho ontológico primitivo. En la concepción que aquí se presenta, la conexión entre universal y particular viene dada por un tropo que es aquello en que precisamente consiste la instanciación del universal en el particular. Hay tantas instanciaciones como universales, particulares y tiempos. Hablar, entonces, de una relación general de 'instanciación' es simplemente hablar de la categoría general de los tropos - pues aquí todo tropo debe ser esencialmente la instanciación de un universal preciso.

Sucede, además, que en una concepción como la que se presenta no existiría ningún problema en identificar un estado de cosas con la suma mereológica de sus componentes. El motivo fundamental por el que habitualmente se ha rechazado tal identificación es que dos estados de cosas $\left[R x_{1} x_{2} A\right.$ y $\left[R x_{2} x_{1} A\right.$ que poseen los mismos componentes son completamente distintos si es que la relación $\mathrm{R}$ que los compone es asimétrica. Podría, entonces existir $\left[R x_{1} x_{2} t\right.$, por ejemplo, y no existir $\left[R x_{2} x_{1} t\right]$, tal como se indicó arriba. Si se introduce un tropo $R$-en- $x_{1}, x_{2}, t$, entonces no hay ninguna posibilidad de que la suma mereológica de los componentes de $\left[R x_{1} x_{2} t\right]$ deje de seleccionar exactamente este estado de cosas y no $\left[R x_{2} x_{1} t\right]$. Si existe el estado de cosas $\left[R x_{1} x_{2} A\right.$, entonces existe la suma mereológica de $R, x_{1}, x_{2}, t$ y $R$-en- $x_{1}, x_{2}, t$. Si existe esta suma mereológica, entonces existe el estado de cosas $\left[R x_{1} x_{2} t\right.$. El tropo R-en- $x_{1}, x_{2}, t$ tiene inscrito -por decirlo de algún modo- en su propia naturaleza que $x_{1}$ tiene la relación $R$ con $x_{2}$ y no al revés. Alguna vez se han manifestado dudas respecto a la inteligibilidad de los estados de cosas porque no poseen una forma de estructuración mereológica (cf. Lewis, 1998). Si los estados de cosas se encuentran fundados en sus componentes del modo que aquí se ha indicado, estas dudas carecen de motivación.

\section{4. ¿Por qué deben estar fundados los estados de cosas?}

Tal como se ha visto, hay un motivo importante para aceptar la existencia de tropos - con las características indicadas- si es que uno admite: (i) la existencia de estados de cosas conformados por universales y objetos particulares, cualquiera sea la naturaleza postulada de esos universales y de esos objetos particulares; y (ii) que los estados de cosas se encuentran fundados en sus componentes. La tesis que ha sido defendida hasta este momento es, por lo tanto, un condicional. Si los estados de cosas se encuentran fundados, entonces hay 
tropos. Uno podría aquí preguntarse de una manera más general, sin embargo, si hay razones para defender de manera independiente la premisa (ii). La justificación independiente de la premisa (i) estará constituida por las razones aducidas para postular universales, las que son ampliamente conocidas. No requiere, por lo tanto, una consideración especial.

Uno podría, en primer lugar, sostener que los estados de cosas deben estar fundados en sus componentes porque toda entidad debe estar fundada en algo. Recuérdese que la relación de fundación es irreflexiva, asimétrica y transitiva, de acuerdo a los principios (5) a (7) formulados arriba. ${ }^{11} \mathrm{Nada}$ puede estar fundado en sí mismo. Por transitividad, si algo estuviese fundado en otra entidad que, a su vez, estuviese fundada en lo primero, entonces algo estaría fundado en sí mismo. No puede haber, por lo tanto, estructuras circulares de fundación. Sostener que todo debe estar fundado en algo es, por esto, sostener que deben existir cadenas infinitas de fundación. Implica sostener también que hay infinitas entidades para entrar en tales cadenas infinitas de fundación. Si, de manera general, todo debe estar fundado en algo, los estados de cosas deben estar fundados en algo. Los estados de cosas se encuentran fundados, tal como se ha explicado, en sus componentes. Esta forma de defensa requiere, adicionalmente, sostener que los componentes de un estado de cosas deban encontrarse, a su vez, fundados en otras entidades, las que, luego, también deben encontrarse fundadas en otras entidades. Y así al infinito. Esto parece, sin embargo, excesivo. Alguna u otra entidad debe resultar fundamental, esto es, no fundada en otras entidades. No sólo se trata de que resulte difícil de entender una cadena infinita de relaciones de fundación. Cualquier ontología debe proponer entidades fundamentales que estén en la base de las relaciones de fundación. La posición que se ha presentado es neutral respecto de qué relaciones ulteriores de fundación puedan darse entre objetos, universales, tiempos y tropos. Eventualmente los tiempos estarán fundados en otras entidades. Eventualmente

${ }^{11}$ La relación de fundación débil, en cambio, es reflexiva y simétrica. Es trivial que todo está fundado débilmente en algo, porque todo está fundado débilmente en sí mismo. Una tesis de este tipo no serviría para justificar la fundación de los estados de cosas, pues sería necesario que los estados de cosas estuviesen, a su vez, fundados en sus componentes. 
lo estarán los objetos. En cualquier caso, una u otra de estas entidades debe resultar fundamental, no fundada en otras. ${ }^{12}$

Si esta vía de justificación del carácter fundado de los estados de cosas no parece razonable, tal vez sea conveniente considerar con más cuidado la posición alternativa a la defendida aquí en donde los estados de cosas no están fundados. Buena parte de las posiciones que se han adoptado en relación con el regreso de Bradley podrían ser formuladas como sosteniendo que los estados de cosas son fundamentales. Recuérdese que una entidad se dice "fundamental" si y sólo si no está fundada en nada (cf. (2)). Si los estados de cosas son fundamentales, entonces no hay nada en lo que estén fundados. Esto podría tomarse, en principio, como compatible con que el estado de cosas dependa ontológicamente de sus componentes. En efecto, tal como se ha destacado ya, las condiciones de identidad de un estado de cosas vienen dados por las entidades de que está compuesto. Si

${ }^{12}$ A pesar de lo extraño que pueda parecer, en la discusión reciente acerca del regreso de Bradley se ha sostenido que la lección que cabe sacar de él es que hay estructuras infinitas de 'análisis' o 'explicación' que resultan satisfactorias (cf. Gaskin, 2008, 354-355; Orilia, 2009). Aquí, tal como para otras discusiones, será clarificador transponer estas formulaciones en términos de relaciones de fundación. El problema planteado por Bradley es que un estado de cosas, por ejemplo [Pat no se encuentra fundado en sus componentes. El estado de cosas [ $I^{1}$ Pat $]$ tampoco se encuentra fundado en sus componentes, ni los estados de cosas $\left[I^{2} I^{1} \mathrm{Pat}\right]$, ó $\left[I^{3} I^{2} I^{1} \mathrm{Pat}\right]$, etcétera. Lo que se ha sostenido, sin embargo, es que aunque ninguno de esos estados de cosas de sucesivos niveles de complejidad están fundados en sus componentes, si se considera la totalidad de todos los infinitos componentes involucrados en tal secuencia sí existiría tal fundación. El estado de cosas [Pat] estaría fundado en $I^{1}, I^{2}, \ldots, I^{\mathrm{n}}, P, a, t$. Aquí, la secuencia $I^{1}, I^{2}, \ldots, I^{\mathrm{n}}$ son las infinitas ocurrencias recursivas de la misma relación de instanciación, o son infinitas relaciones de instanciación diferentes para infinitos tipos lógicos diferentes. El 'paso' al infinito propuesto por Gaskin y Orilia puede hacer vacilar nuestras intuiciones inicialmente, pero subsisten aquí problemas cruciales. En primer lugar, no es nada de claro que exista una 'totalidad' de infinitas relaciones de instanciación de distintos tipos lógicos, o que existan infinitas ocurrencias diferentes de la misma relación de instanciación. Más bien, la situación parece ser que no hay tal totalidad. En segundo lugar, no se ve porqué la existencia de la secuencia infinita $I^{1}, I^{2}, \ldots$, $I^{\mathrm{n}}$ implique aquí alguna diferencia. Las infinitas relaciones de instanciación, o bien la relación de instanciación de la que se espera que esté ocurriendo recursiva e infinitamente podrían existir y no existir el estado de cosas [Pat]. 
los estados de cosas son entidades fundamentales, entonces su existencia debe ser, de algún modo, un hecho ontológico 'primitivo'. Una alternativa teórica de este tipo es la que ha sido propuesta por filósofos como Herbert Hochberg (cf. Hochberg, 1978, 338-339). También, sin embargo, es ésta la alternativa que uno podría adscribir a quienes sostienen que la instanciación es un hecho primitivo (cf. Strawson, 1959, 167-168; Bergmann, 1967, 9-12; Armstrong, 1978a, 108-113; 1989, 108-110; 1997, 127; 2004, 46-48). En efecto, al sostener que no debe suponerse que la instanciación sea una relación como cualquier otra - con lo que se dispara el regreso de Bradleyse está sosteniendo que no debe tratar de fundarse el hecho de que un universal esté instanciado en ciertos universales en cierto tiempo en alguna entidad adicional a esos universal, objetos y tiempo. Los estados de cosas son simplemente entidades fundamentales. Un estado de cosas es esencialmente el instanciarse de un universal en uno o varios objetos en un tiempo. Si este hecho es fundamental -llámese "instanciación" o como se quieraentonces los estados de cosas son entidades fundamentales.

Hay motivos, sin embargo, para pensar que los estados de cosas no son fundamentales del modo indicado. Como la contradictoria de esta tesis es precisamente que los estados de cosas están fundados, y como la mejor alternativa para fundar los estados de cosas es suponer que hay tropos, estos serían motivos para pensar que hay tropos. Un primer motivo para sostener que los estados de cosas no pueden ser fundamentales es porque se encuentran fundados parcialmente en sus componentes. La tesis general que podría sugerirse aquí es que todo lo que está fundado parcialmente está también fundado. Algo así es lo que parece imponer la definición de fundación parcial dada arriba (cf. (9)): [( $x$ está fundado parcialmente en $y)={ }_{\mathrm{df}} \exists z_{1} z_{2}$ $\ldots z_{n}\left(x\right.$ está fundado en $\left.\left.y, z_{1}, z_{2}, \ldots, z_{n}\right)\right]$. Como se ha identificado la dependencia ontológica con la fundación parcial, el hecho de que los estados de cosas dependan ontológicamente de los universales, objetos y tiempos que los componen -algo difícilmente cuestionable, dado que las condiciones de identidad de un estado de cosas están constituidas por tales componentesse sigue que los estados de cosas deben estar fundados. Este argumento, sin embargo, no parece muy verosímil. En primer lugar, no parece haber una justificación independiente para la tesis general aparte de la forma en que ha sido definida la 'fundación parcial' en términos de la 'fundación'. Esto parece una forma de obtener la fundación de los estados de cosas por mera estipulación y, lo que es más, obtener también por definición tropos, ya 
que deben postularse tropos si es que los estados de cosas están fundados. En segundo lugar, si se admite la tesis general según la que todo lo que está fundado parcialmente o depende ontológicamente de otra entidad, está también fundado, se genera un problema estructuralmente semejante para los tropos que están aquí siendo postulados. En efecto, los tropos que se han postulado para la fundación de los estados de cosas dependen ontológicamente del universal del que son la instanciación, del objeto u objetos en donde se instancian y del tiempo cuando se instancian. Hay aquí un margen importante para variaciones acerca de cómo hayan de ser entendidos los objetos y los tiempos, pero comoquiera que sean entendidos, los tropos serán ontológicamente dependientes de ellos. Si la dependencia ontológica es fundación parcial, entonces debería sostenerse que estos tropos deben estar fundados. El universal del que sean instanciación, junto con el objeto u objetos y el tiempo en donde se instancian no serían fundamento de tales tropos. Se generaría, entonces, un nuevo regreso de Bradley. La postulación de tales tropos como solución al regreso resultaría completamente inútil.

Hay un segundo motivo, sin embargo, para rechazar el carácter fundamental de los estados de cosas mucho más convincente. En efecto, la instanciación primitiva de un universal en uno o varios objetos en un tiempo parece diferir de un tropo sólo en el nombre. En efecto, quien postula un tropo que esencialmente es la instanciación de un universal en uno o varios objetos en un instante de tiempo está postulando una entidad particular cuya naturaleza se agota en ser la 'conexión' de tales universal, objetos y tiempo. ¿Qué otra cosa sería un hecho de 'instanciación primitiva’? Quien sostenga que los estados de cosas son entidades fundamentales, dado que las instanciaciones son hechos ontológicos primitivos, estará postulando como entidades fundamentales a tales estados de cosas, los universales y los objetos -si se dejan a un lado los tiempos. Son tres tipos de entidades fundamentales. Quien postula un tropo que funda el estado de cosas estará postulando también tres tipos de entidades fundamentales: universales, objetos y tropos. Existe aquí, sin embargo, la ventaja teórica de que en vez de suponer que hay un hecho ontológico primitivo de 'instanciación', una relación que no es relación, se admite una categoría ontológica perfectamente conocida y largamente estudiada. Esto deja abierta la posibilidad, además, de que los objetos sean entendidos como cúmulos de tropos, con lo que los tipos de entidades fundamentales en la ontología se reducirían a dos: universales y tropos. 
Así, resulta que, o bien los estados de cosas están fundados en sus constituyentes o bien no lo están. En el primer caso, se debe postular un tropo que esencialmente es la instanciación de un universal en uno o varios objetos en un tiempo. En el segundo caso, se debe postular una categoría ontológica adicional y hechos ontológicamente primitivos que parecen poco económicos y que, además, parecen diferir de un tropo sólo en el nombre. Estos son motivos para pensar que los estados de cosas están, efectivamente, fundados en sus componentes y que hay tropos.

\section{Conclusiones}

Se ha sostenido en este trabajo que, si los estados de cosas se encuentran fundados en sus constituyentes, entonces deben postularse tropos con ciertas características específicas. Para esto, se ha considerado el conocido problema del 'regreso de Bradley' como un problema acerca de la -eventual- relación de fundación entre un estado de cosas y sus componentes. La relación de fundación es una relación primitiva, multígrada, irreflexiva, asimétrica, transitiva y que determina un patrón modal entre diferentes entidades. Parece la forma más prometedora para considerar las cuestiones que tradicionalmente se han tratado como cuestiones de 'reducción', 'superveniencia' o 'explicación ontológica'. Un estado de cosas es una estructuración -en principio, no mereológica- de universales, objetos y tiempos. Cualquier ontología que postule universales debe explicar -ulteriormentecómo es que tal universal se 'conecta' con un objeto particular y, con ello, cómo es que se constituye un estado de cosas.

El problema del regreso de Bradley es una dificultad sistemática que tiene que ver, precisamente, con la conformación de tales estructuras. La existencia del universal que integra un estado de cosas, el objeto u objetos que integran un estado de cosas, y el tiempo que integra un estado de cosas, no son suficientes para garantizar la existencia de éste. El estado de cosas no parece estar fundado en sus componentes. Si uno aquí introduce una relación de 'instanciación’ entre universal y particular, la situación no mejora, pues tal relación de instanciación es un universal como cualquier otro y podría existir sin estar, de hecho, instanciado, en tales universal, objeto u objetos, y tiempo. Otras relaciones ulteriores de instanciación tampoco mejoran la situación. Si uno, en cambio, introduce una relación particular de instanciación, que esencialmente sea la instanciación del universal en cues- 
tión en el objeto u objetos en cuestión, en el tiempo en cuestión, el estado de cosas sí estará fundado en sus componentes. Es más. No habría ninguna dificultad para sostener que un estado de cosas es aquí la fusión mereológica de sus componentes.

Al examinar, entonces, el problema del regreso de Bradley como un problema acerca de la fundación de un estado de cosas en sus constituyentes, aparece que si los estados de cosas están fundados en sus constituyentes, hay tropos. Una cuestión ulterior, sin embargo, es si hay o no motivos para pensar que los estados de cosas deben estar fundados. No parece razonable, en efecto, sostener que toda entidad deba estar fundada. Algunas estarán fundadas, otras serán fundamentales. Tampoco parece una buena razón para sostener que los estados de cosas están fundados en sus constituyentes que los estados de cosas dependen ontológicamente de sus constituyentes. Si toda entidad que fuese dependiente ontológicamente de otra estuviese fundada, un problema análogo al regreso de Bradley surgiría para los tropos que han sido postulados precisamente para evitar este regreso. Su postulación sería inútil.

Si se sostiene que los estados de cosas son fundamentales, esto es, no están fundados en nada, deben postularse hechos ontológicos primitivos que podrían ser evitados. En este respecto, la fundación de los estados de cosas en sus componentes que incluyen tropos, ofrece ventajas teóricas. ${ }^{13}$

\section{Referencias bibliográficas}

David M. Armstrong (1978a), Universals and Scientific Realism, Volume I: Nominalism and Realism, Cambridge: Cambridge University Press.

David M. Armstrong (1978b), Universals and Scientific Realism, Volume II: A Theory of Universals, Cambridge: Cambridge University Press.

David M. Armstrong (1989), Universals. An Opinionated Introduction, Boulder: Westview.

David M. Armstrong (1997), A World of States of Affairs, Cambridge: Cambridge University Press.

${ }^{13}$ Este trabajo ha sido redactado en ejecución del proyecto de investigación Fondecyt 1120015 (Conicyt, Chile). Una versión preliminar fue presentada en el seminario del Instituto de Filosofía de la Pontificia Universidad Católica de Chile. Agradezco las sugerencias y comentarios de los asistentes. 
David M. Armstrong (2004), Truth and Truthmakers, Cambridge: Cambridge University Press.

Paul Audi (2012), "A Clarification and Defense of the Notion of Grounding” en Correia y Schnieder (2012a), 101-121.

Gustav Bergmann (1967), Realism. A Critique of Brentano and Meinong, Madison, Milwaukee: The University of Wisconsin Press.

Francis H. Bradley (1897), Appearance and Reality, Oxford: Clarendon Press. Keith Campbell (1990), Abstract Particulars, Oxford: Blackwell.

Fabrice Correia y Benjamin Schnieder (eds.) (2012a), Metaphysical Grounding. Understanding the Structure of Reality, Cambridge: Cambridge University Press. Fabrice Correia y Benjamin Schnieder (2012b), "Grounding: An Opinionated Introduction” en Correia y Schnieder (2012a), 1-36.

Fabrice Correia (2012), "On the Reduction of Necessity to Essence" Philosophy and Phenomenological Research 84, 639-653.

Douglas Ehring (2011), Tropes. Properties, Objects, and Mental Causation, Oxford: Oxford University Press.

Kit Fine (1994), "Essence and Modality" Philosophical Perspectives 8, 1-16.

Kit Fine (1995), "Ontological Dependence" Proceedings of the Aristotelian Society 95, 269-290.

Kit Fine (2010), "Some Puzzles of Ground" Notre Dame Journal of Formal Logic 51, 97-118.

Kit Fine (2012a), "The Pure Logic of Ground" The Review of Symbolic Logic 5, 1-25.

Kit Fine (2012b), "Guide to Ground" en Correia y Schnieder (2012a), 37-80.

Richard Gaskin (2008), The Unity of the Proposition, Oxford: Oxford University Press.

Reinhardt Grossmann (1973), Ontological Reduction, Bloomington: Indiana University Press.

Reinhardt Grossmann (1983), The Categorial Structure of the World, Bloomington: Indiana University Press.

John Heil (2003), From an Ontological Point of View, Oxford: Clarendon Press. John Heil (2012), The Universe as We Find it, Oxford: Clarendon Press. Herbert Hochberg (1978), Thought, Fact, and Reference. The Origins and Ontology of Logical Atomism, Minneapolis: University of Minnesota Press.

Kathrin Koslicki (2012), "Essence, Necessity, and Explanation” en Tuomas 
E. Tahko (ed.), Contemporary Aristotelian Metaphysics, Cambridge: Cambridge University Press, 187-206.

David Lewis (1991), Parts of Classes, Oxford: Blackwell.

David Lewis (1998), "A World of Truthmakers?”, Times Literary Supplement 4950 (13 de febrero de 1998), 30. Reimpreso en Lewis (1999), 215-220. Se cita por esta última versión.

David Lewis (1999), Papers in Metaphysics and Epistemology, Cambridge: Cambridge University Press.

E. Jonathan Lowe (2006), The Four-Category Ontology. A Metaphysical Foundation for Natural Science, Oxford: Clarendon Press.

Charles B. Martin (1980), "Substance Substantiated", Australasian Journal of Philosophy 58, 3-10.

Anna-Sofia Maurin (2002), If Tropes, Dordrecht: Kluwer.

Anna-Sofia Maurin (2011), "An Argument for the Existence of Tropes" Erkenntnis 74, 69-79.

Bo Meinertsen (2008), "A Relation as the Unifier of States of Affairs" Dialectica 62, 1-19.

D. W. Mertz (1996), Moderate Realism and its Logic, New Haven: Yale University Press.

Francesco Orilia (2009), "Bradley's Regress and Ungrounded Dependence Chains: A Reply to Cameron” Dialectica 63, 333-341.

Gideon Rosen (2010), "Metaphysical Dependence: Grounding and Reduction" en Bob Hale and Aviv Hoffman (eds.), Modality: Metaphysics, Logic, and Epistemology, Oxford: Oxford University Press, 109-136.

Gonzalo Rodriguez-Pereyra (2002), Resemblance Nominalism. A Solution to the Problem of Universals, Oxford. Clarendon Press.

Jonathan Schaffer (2009), "On What Grounds What" en David Chalmers, David Manley y Ryan Wasserman (eds.), Metametaphysics. New Essays on the Foundations of Ontology, Oxford: Clarendon Press, 347-383.

Benjamin Schnieder (2011), "A Logic for 'Because" The Review of Symbolic Logic 4, 445-465.

Peter Simons (1987), Parts. A Study in Ontology, Oxford: Clarendon Press. Peter Simons (1994), "Particulars in Particular Clothing: Three Trope Theories of Substance", Philosophy and Phenomenological Research 54, 553-575.

Peter F. Strawson (1959), Individuals. An Essay in Descriptive Metaphysics, London: Methuen. 
William Vallicella (2000), "Three Conceptions of States of Affairs", Noûs 34, 237-259.

William Vallicella (2002), "Relations, Monism, and the Vindication of Bradley's Regress", Dialectica 56, 3-35.

Jan Willem Wieland y Arianna Betti (2008), "Relata-Specific Relations: A Response to Vallicella", Dialectica 62, 509-524.

Donald C. Williams (1953a), "On the Elements of Being: I", The Review of Metaphysics 7, 3-18.

Donald C. Williams (1953b), "On the Elements of Being: II”, The Review of Metaphysics 7, 71-92.

Recibido: 4/2014; aceptado: 7/2014 